\title{
ESTADO NUTRICIONAL E QUALIDADE DE VIDA EM ADULTOS E IDOSOS COM DEPRESSÃO
}

\section{Nutritional status and quality of life in adults and older adults with depression}

Estado nutricional y calidad de vida de adultos y mayores con depresión

\author{
Aline Rodrigues Godoy (iD \\ Universidade do Vale do Taquari - UNIVATES - Lajeado (RS) - Brasil \\ Fernanda Scherer Adami iD \\ Universidade do Vale do Taquari - UNIVATES - Lajeado (RS) - Brasil
}

\begin{abstract}
RESUMO
Objetivo: Avaliar o estado nutricional e a qualidade de vida em adultos e idosos com e sem depressão. Métodos: Estudo quantitativo e transversal, com 79 usuários das Unidades Básicas de Saúde de municípios do Vale do Taquari, Rio Grande do Sul. Para determinar os escores dos domínios (físico, psicológico, ambiental e social) de qualidade de vida (QV) foi aplicado o questionário World Health Organization Quality of Life-Bref e um questionário socioeconômico (idade, renda, escolaridade e estado civil). A avaliação antropométrica contemplou peso, estatura, Índice de Massa Corporal, Circunferência da Cintura (CC) e Circunferência do Pescoço (CP). O diagnóstico de depressão foi realizado por psicólogas. O nível de significância máximo assumido foi $5 \%(p \leq 0,05)$, análise realizada através do software SPSS versão 22.0. Utilizaram- se os testes Mann-Whitney, correlação de Pearson e qui-quadrado. Resultados: Verificou-se correlação direta entre idade e domínio físico $(p=0,017), p s i ́ q u i c o ~(p<0,01)$, social $(p=0,001)$ e ambiental $(p=0,003)$. Os homens obtiveram média superior e significativa no domínio ambiental $(p=0,009)$ em relação às mulheres. Os pacientes sem diagnóstico de depressão apresentaram média significativamente superior nos domínios físico ( $p=0,035)$ e psicológico ( $p=0,042)$. Em relação ao estado nutricional, a magreza e eutrofia apresentaram média superior no domínio físico $(p=0,015)$. Conclusão: As melhores percepções sobre os domínios de QV foram observadas entre os homens, idosos e participantes sem diagnóstico de depressão. Verificou-se correlação direta entre a idade e todos os domínios e que os participantes depressivos eram, em sua maioria, obesos, com risco cardiovascular e sem risco de excesso de peso.
\end{abstract}

Descritores: Estado Nutricional; Qualidade de Vida; Depressão.

\section{ABSTRACT}

Objective: To assess nutritional status and quality of life in adults and older adults with and without depression. Methods: This is a quantitative cross-sectional study of 79 users of Primary Health Care Centers located in the municipalities in the Vale do Taquari, Rio Grande do Sul. To determine the scores of the Quality of Life (QoL) domains (physical, psychological, environmental and social) we used the World Health Organization Quality of Life-Bref questionnaire and a socioeconomic questionnaire (age, income, education and marital status). Anthropometric measurement included weight, height, Body Mass Index, Waist Circumference (WC) and Neck Circumference (NC). Diagnosis of depression was given by psychologists. The maximum significance level assumed was $5 \%(p \leq 0.05)$ and the analysis was performed using SPSS version 22.0. The Mann-Whitney test, the Pearson's correlation coefficient and the Chi-squared test were used. Results: There was a direct correlation between age and the physical ( $p=0.017)$, psychological $(p<0.01)$, social $(p=0.001)$ and environmental $(p=0.003)$ domains. Men scored significantly higher in the environmental domain $(p=0.009)$ compared with women. Patients without a diagnosis of depression scored significantly higher in the physical $(p=0.035)$ and psychological $(p=0.042)$ domains. As for nutritional status, thinness and normal weight were associated with higher mean scores in the physical domain $(p=0.015)$. Conclusion: The best perceptions of QoL domains were observed among men, older adults and participants without a diagnosis of depression. There was a direct correlation between age and all the domains, and depressive participants were mostly obese and presented cardiovascular risk, but without risk of presenting excess weight.

Descriptors: Nutritional Status; Quality of Life; Depression.

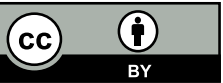




\section{RESUMEN}

Objetivo: Evaluar el estado nutricional y la calidad de vida de adultos y mayores con y sin depresión. Métodos: Estudio cuantitativo y transversal con 79 usuarios de las Unidades Básicas de Salud de los municipios del Vale de Taquari, Rio Grande do Sul. Se ha aplicado el cuestionario World Health Organization Quality of Life-Bref y un cuestionario socioeconómico (edad, renta, escolaridad y estado civil) para determinar las puntuaciones de los dominios (físico, psicológico, ambiental y social) de la calidad de vida (CV). En la evaluación antropométrica se ha incluido el peso, la estatura, el Índice de Masa Corporal, la Circunferencia de la Cintura (CC) e la Circunferencia del Cuello (CC). El diagnóstico de depresión ha sido realizado por psicólogas. El nivel de significación máxima asumido ha sido del $5 \%(p \leq 0,05)$ y el análisis ha sido realizado a través del software SPSS versión 22.0. Se utilizaron las pruebas de Mann-Whitney, la correlación de Pearson y el chi-cuadrado. Resultados: Se verificó la correlación directa entre la edad y el dominio físico $(p=0,017)$, el psíquico $(p<0,01)$, el social $(p=0,001)$ y el ambiental $(p=0,003)$. Los hombres tuvieron la media superior y significativa para el dominio ambiental $(p=0,009)$ en comparación a las mujeres. Los pacientes sin el diagnostico de depresión presentaron la media significativamente superior para los dominios físico $(p=0,035)$ y el psicológico $(p=0,042)$. Respecto el estado nutricional, la delgadez y la eutrofia presentaron media superior para el dominio físico $(p=0,015)$. Conclusión: Las mejores percepciones de los dominios de CV han sido observadas entre los hombres, los mayores y los participantes sin el diagnostico de depresión. Se verificó la correlación directa entre la edad y todos los dominios y que los participantes depresivos eran, en su mayoría, obesos, con riesgo cardiovascular y sin riesgo de exceso de peso.

Descriptores: Estado Nutricional; Calidad de Vida; Depresión.

\section{INTRODUÇÃO}

A depressão teve seus primeiros registros datados 500 anos antes de Cristo(1). Ela é considerada um transtorno mental grave, com sintomas associados à diminuição da autoestima, alterações no sono e apetite, cansaço, sentimentos de ineficácia, diminuição no interesse ou prazer e falta de concentração(2). A depressão é considerada uma das principais causas de problemas de saúde e de incapacidade no mundo. A Organização Mundial da Saúde (OMS) estima que mais de 322 milhões de pessoas possuem essa doença, considerando o aumento de $18 \%$ nos casos diagnosticados entre os anos de 2005 e 2015. O transtorno mental pode levar ao suicídio, que é a segunda principal causa de morte entre pessoas com idade entre 15 e 29 anos. Anualmente, cerca de 800 mil pessoas morrem por suicídio(3).

A OMS evidencia que a falta de apoio às pessoas com transtornos mentais, o medo do estigma social e a falta de recursos e de profissionais capacitados são obstáculos para que muitas pessoas tenham ou busquem acesso ao tratamento necessário para manter suas vidas saudáveis ${ }^{(4)}$. Menos da metade das pessoas recebem o tratamento

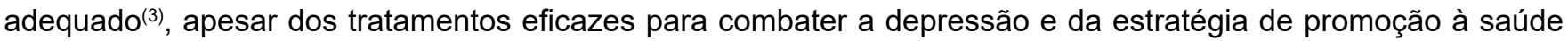
do Sistema Único de Saúde (SUS), cujo enfoque está no processo de saúde-adoecimento no Brasil e nas suas possíveis formas de intervenção( ${ }^{(5)}$.

O aumento da qualidade de vida de pessoas com os transtornos depressivos se dá pela redução das desigualdades sociais em saúde, o que ativa os gastos do sistema em questão, quando associado à oferta de serviços de saúde mental e às ações educativas ${ }^{(6)}$. O oferecimento de serviços em uma Unidade Básica de Saúde (UBS) é feito por uma equipe multiprofissional, que é responsável por um conjunto de condutas de âmbito individual ou coletivo, com interesse de promover a proteção à saúde, tanto na precaução de agravos quanto na diminuição dos danos, no diagnóstico, na intervenção do tratamento e na manutenção da saúde, com o intuito de desenvolver atenção integral que impacte na situação de saúde e na autonomia dos usuários ${ }^{(7)}$.

A qualidade de vida (QV) de uma população é relacionada diretamente às suas vivências e aos seus acessos a alguns sistemas econômicos e sociais, como emprego e renda, educação básica, alimentação adequada, bons serviços de saúde, saneamento básico, habitação e transporte, considerando a definição dos índices de $Q V^{(8)}$.

A saúde possui um amplo conceito e, por isso, engloba a pessoa nas suas inúmeras dimensões. Desse modo, ela está diretamente ligada ao modo de viver dos indivíduos, que é motivado pelo conhecimento, pelo ambiente físico e pelos contextos social, econômico e cultural de cada cidadão(9). Além disso, essa concepção está atrelada ao bem-estar, pois as pessoas mencionam ter saúde quando se sentem bem e são capazes de realizar suas atividades diárias ${ }^{(10)}$, portanto assegurar o acesso democrático aos serviços de saúde é um dos principais desafios do setor público, principalmente em países que apresentam desmandas crescentes provocadas pelo aumento da expectativa de vida e longevidade ${ }^{(11)}$.

O presente estudo tem como objetivo avaliar o estado nutricional e a qualidade de vida em adultos e idosos com e sem depressão atendidos em Unidades Básicas de Saúde (UBS). 


\section{MÉTODOS}

Trata-se de estudo quantitativo, transversal e analítico, desenvolvido nas UBS dos Municípios de Travesseiro, Marques de Souza e Arroio do Meio, todos pertencentes à região do Vale do Taquari, estado do Rio Grande do Sul, Brasil. Estes municípios foram selecionados por realizarem atendimento psicológico na UBS. A amostragem se deu por conveniência. Inicialmente, os profissionais Psicólogos convidaram seus pacientes de ambos os sexos, maiores de 18 anos, com atendimento psicológico nas UBS, a participarem do estudo e a assinarem o Termo de Consentimento Livre Esclarecido (TCLE). Excluíram-se os pacientes que não compareceram no dia agendado para coleta de dados ou que por algum motivo desistiram da participação.

Dos 200 pacientes atendidos no período de 2015 a 2016, 79 aceitaram participar, entre homens e mulheres, com idades entre 28 a 79 anos, com e sem diagnóstico de depressão. Os participantes do estudo foram categorizados como depressivos e não depressivos pelo profissional Psicólogo da UBS.

Após a seleção dos participantes, aplicou-se o questionário World Health Organization Quality of Life (WHOQOLBREF) composto por 26 questões, com ênfase em quatro domínios de qualidade de vida: físico, psicológico, de ambiente e social ${ }^{(12)}$. Trata-se de uma versão abreviada do World Health Organization Quality of Life-100 (WHOQOL-100), que foi primeiramente desenvolvido para idosos, porém validado e traduzido para o português com características abrangentes e de simples aplicabilidade, visto que cada domínio é composto por questões, cujas pontuações das respostas variam entre 1 e $5^{(13)}$. As médias dos cálculos são escores finais de cada domínio, as quais consideram as respostas das questões, gerando pontuações em uma escala de 4 a $20^{(14)}$, sendo que os escores de cada domínio do questionário WHOQOL-BREF foram transformados em escalas de 0 a 100, considerando que a melhor qualidade de vida é atribuída para aqueles que conseguirem o maior escore ${ }^{(12)}$.

O domínio físico engloba questões sobre dor e desconforto, energia e fadiga, sono, mobilidade, atividades diárias, uso de medicação e capacidade para o trabalho. No domínio psicológico, os quesitos são sobre sentimentos, aprendizagem, memória, concentração, autoestima, aparência e religião. O domínio meio ambiente apresenta questionamentos relacionados à segurança e proteção, recursos financeiros, possibilidade de aquisição de novas informações e habilidades, atividades de lazer, trânsito e transporte. Por fim, o domínio relações sociais aborda as relações familiares e de vida social, além de atividade sexual(15).

Em seguida, aplicou-se um questionário estruturado, composto por questões fechadas sobre sexo, idade, renda, escolaridade e estado civil. A avaliação antropométrica contemplou peso, estatura, Índice de Massa Corporal (IMC), sendo o instrumento classificado para adultos, de acordo com a Organização Mundial de Saúde (OMS)(16) , e para idosos, segundo a Organização Pan-Americana de Saúde (OPAS) ${ }^{(17)}$. Os adultos e idosos foram agrupados segundo seu estado nutricional e sua faixa etária. Para realizar a circunferência da cintura (CC), orientou-se o participante a ficar em pé e a medida foi localizada na região abdominal, em seu menor perímetro, realizada no momento de expiração, sendo que o ponto de corte para classificação de risco de doenças cardiovasculares mensurado como igual ou maior a $94 \mathrm{~cm}$ para homens e a $80 \mathrm{~cm}$ para as mulheres. Para circunferência do pescoço (CP), o participante foi orientado a ficar em pé, sendo a medida aferida na altura média do pescoço, cuja mensuração para o ponto de corte para seleção era igual ou maior a $37 \mathrm{~cm}$ para homens e maior do que $34 \mathrm{~cm}$ para mulheres. Medidas abaixo desses valores, observando-se a CP, foram consideradas sem risco de excesso de peso(17).

Para a coleta de dados referente à avaliação antropométrica utilizou-se uma balança digital portátil da marca Plenna $^{\circledR}$, com capacidade máxima de $180 \mathrm{Kg}$ e com precisão de $100 \mathrm{~g}$. O estadiômetro portátil profissional Sanny ${ }^{\circledR}$, com precisão de um milímetro, foi utilizado para aferir a medida da estatura. Para averiguar as circunferências da cintura e do pescoço, utilizou-se fita métrica inelástica, da marca Cardiomed $^{\circledR}$, com medida máxima de $150 \mathrm{~cm}$.

Os resultados foram considerados significativos em um nível de significância máximo de $5 \%$ ( $\leq \leq 0,05)$, utilizandose o software o Statistical Package for the Social Sciences, versão 22.0 para a análise dos dados, por meio dos testes de Mann-Whitney, da análise de correlação de Pearson e do teste qui-quadrado.

O estudo recebeu aprovação pelo Comitê de Ética em Pesquisa da Universidade do Vale do Taquari - UNIVATES, sob Parecer $n^{0} 1.230 .745$.

\section{RESULTADOS}

Observou-se que a maioria da população estudada era do sexo feminino $75,9 \%$ ( $n=60)$, adulto $55,7 \%$ ( $n=44)$ e sem depressão $51,9 \%(n=41)$. Apresentaram média de estatura de $1,61 \mathrm{~m}( \pm 0,08)$, peso de $76,45 \mathrm{Kg}( \pm 16,52)$, Índice de Massa Corporal de $29,35 \mathrm{Kg} / \mathrm{m}^{2}( \pm 5,80)$, CC $90 \mathrm{~cm}( \pm 12,27)$ e CP $33,41 \mathrm{~cm}( \pm 3,32)$. 
Em relação aos domínios de qualidade de vida, verificou-se que a média do domínio físico foi de $54,75( \pm 19,58)$, a média do domínio psíquico de $56,65( \pm 17,52)$, a do domínio social de $67,72( \pm 16,36)$ e a do domínio ambiental de $61,75( \pm 12,45)$.

A maior parte da população estudada relatou possuir renda familiar entre 1 e 2 salários mínimos (84,8\%; $n=67)$, ensino fundamental incompleto $(75,9 \%$; $n=60)$, estado civil casado e/ou união estável $(68,4 \% ; n=54)$, classificação do estado nutricional de obesidade $(40,5 \% ; n=32)$, classificação pela CC como em risco de doenças cardiovasculares $(67,1 \% ; n=53)$ e sem risco de excesso de peso pela CP $(69,6 \% ; n=55)$ (Tabela I).

Tabela I - Caracterização socioeconômica e antropométrica dos adultos e idosos avaliados nas Unidades Básicas de Saúde dos municípios do Vale do Taquari. Rio Grande do Sul, Brasil, 2017.

\begin{tabular}{llcc}
\hline Variável & Resposta & $\mathbf{n}$ & $\%$ \\
\hline Renda & Menos de 1 salário mínimo & 11 & 13,9 \\
& 1 a 2 salários mínimos & 67 & 84,8 \\
Escolaridade & 3 ou mais salários mínimos & 1 & 1,3 \\
& Fundamental Incompleto & 60 & 75,9 \\
& Fundamental Completo & 7 & 8,9 \\
& Médio Incompleto & 2 & 2,5 \\
& Médio Completo & 7 & 8,9 \\
& Superior Incompleto & 2 & 2,5 \\
Estado Civil & Superior Completo & 1 & 1,3 \\
& Solteiro & & 7,6 \\
& Casado/união estável & 6 & 68,4 \\
& Viúvo & 54 & 19,0 \\
Classificação IMC & Separado & 15 & 5,1 \\
& Magreza & 4 & 3,8 \\
& Eutrofia & 3 & 26,6 \\
& Sobrepeso & 21 & 29,1 \\
Classificação CC & Obesidade & 23 & 40,5 \\
& Sem risco cardiovascular & 32 & 32,9 \\
Classificação CP & Com risco cardiovascular & 26 & 67,1 \\
& & 53 & 69,6 \\
\hline & Sem risco excesso de peso & & 30,4 \\
\hline
\end{tabular}

CC: Circunferência da cintura; CP: Circunferência do pescoço; IMC: Índice de Massa corporal

O sexo masculino apresentou média significativamente superior no domínio ambiental $(p=0,009)$. Em relação aos outros domínios, não foi observada diferença significativa entre os sexos, contudo, as médias também foram superiores entre os homens.

Em relação à comparação entre os pacientes com e sem diagnóstico de depressão, os resultados demonstraram média significativamente superior para o domínio físico $(p=0,035)$ e psicológico $(p=0,042)$ entre os pacientes não deprimidos. Para os domínios ambiental $(p=0,102)$ e social $(p=0,066)$ as médias também foram superiores entre os não deprimidos, no entanto, a diferença não se mostrou significativa.

Os idosos apresentaram média significativamente superior em relação aos adultos para os domínios físico $(p=0,006)$, psicológico $(p \leq 0,01)$, social $(p=0,016)$ e ambiental $(p=0,001)$ (Tabela II).

A população com sobrepeso e obesidade apresentou médias significativamente inferiores aos com magreza e eutrofia para o domínio físico $(p=0,015)$. Em relação aos demais domínios, não se observou diferença significativa no quesito estado nutricional. O risco cardiovascular, classificado por meio da CC, e o risco de excesso de peso, dado obtido por meio da CP, obtiveram médias superiores nos participantes sem risco, sendo que não apresentaram resultados significativos em nenhum dos domínios (Tabela III). 
Em relação à idade (Tabela IV), verificou-se correlação direta entre a idade e todos os domínios. No entanto, demonstrou-se correlação inversa entre o IMC e os domínios físico, psíquico e ambiental. Referente à CC e CP, não se observou relação significativa com os domínios.

Tabela II - Comparação entre os domínios de qualidade de vida, com sexo, diagnóstico clínico de depressão e faixa etária de adultos e idosos, nas Unidades Básicas de Saúde dos municípios do Vale do Taquari. Rio Grande do Sul, Brasil, 2017.

\begin{tabular}{|c|c|c|c|c|}
\hline Domínio & Sexo & $\mathbf{n}$ & Média \pm DP & $\mathbf{p}^{*}$ \\
\hline \multirow[t]{2}{*}{ Físico } & Feminino & 60 & $52,80 \pm 18,83$ & 0,146 \\
\hline & Masculino & 19 & $60,90 \pm 21,14$ & \\
\hline \multirow[t]{2}{*}{ Psíquico } & Feminino & 60 & $55,07 \pm 19,23$ & 0,322 \\
\hline & Masculino & 19 & $61,62 \pm 9,17$ & \\
\hline \multirow[t]{2}{*}{ Social } & Feminino & 60 & $65,83 \pm 17,27$ & 0,062 \\
\hline & Masculino & 19 & $73,68 \pm 11,54$ & \\
\hline \multirow[t]{2}{*}{ Ambiental } & Feminino & 60 & $59,74 \pm 13,16$ & 0,009 \\
\hline & Masculino & 19 & $68,09 \pm 6,95$ & \\
\hline Domínio & Diagnóstico & $\mathrm{n}$ & Média \pm DP & $\mathbf{p}^{*}$ \\
\hline \multirow[t]{2}{*}{ Físico } & Deprimido & 38 & $49,25 \pm 19,63$ & 0,035 \\
\hline & Não deprimido & 41 & $59,84 \pm 18,33$ & \\
\hline \multirow[t]{2}{*}{ Psíquico } & Deprimido & 38 & $52,52 \pm 18,11$ & 0,042 \\
\hline & Não deprimido & 41 & $60,47 \pm 16,25$ & \\
\hline \multirow[t]{2}{*}{ Social } & Deprimido & 38 & $64,04 \pm 17,66$ & 0,066 \\
\hline & Não deprimido & 41 & $71,14 \pm 14,45$ & \\
\hline \multirow[t]{2}{*}{ Ambiental } & Deprimido & 38 & $59,70 \pm 11,57$ & 0,102 \\
\hline & Não deprimido & 41 & $63,64 \pm 13,07$ & \\
\hline Domínio & Faixa etária & $\mathrm{n}$ & Média \pm DP & $\mathbf{p}^{*}$ \\
\hline \multirow[t]{2}{*}{ Físico } & Adulto & 44 & $49,03 \pm 21,07$ & 0,006 \\
\hline & Idoso & 35 & $61,94 \pm 14,93$ & \\
\hline \multirow[t]{2}{*}{ Psíquico } & Adulto & 44 & $49,53 \pm 19,36$ & $p<0,01$ \\
\hline & Idoso & 35 & $65,60 \pm 9,06$ & \\
\hline \multirow[t]{2}{*}{ Social } & Adulto & 44 & $63,07 \pm 18,01$ & 0,016 \\
\hline & Idoso & 35 & $73,57 \pm 11,87$ & \\
\hline \multirow[t]{2}{*}{ Ambiental } & Adulto & 44 & $57,32 \pm 13,05$ & 0,001 \\
\hline & Idoso & 35 & $67,32 \pm 9,11$ & \\
\hline
\end{tabular}

* Teste Mann Whitney; DP: desvio padrão

Mesmo não apresentando diferença significativa, os participantes depressivos classificaram-se, em sua maioria, como obesos, com risco cardiovascular em relação à CC e sem risco de excesso de peso em relação à CP. Os não deprimidos apresentaram-se eutróficos, com risco cardiovascular em relação à CC e sem risco de excesso de peso em relação à CP (Tabela $\mathrm{V}$ ). 
Tabela III - Comparação entre os domínios de qualidade de vida com o estado nutricional, de acordo com o Índice de Massa Corporal, o risco de excesso de peso, conforme circunferência do pescoço, e o risco cardiovascular, segundo circunferência da cintura, de adultos e idosos de Unidades Básicas de Saúde de municípios do Vale do Taquari. Rio Grande do Sul, Brasil, 2017.

\begin{tabular}{|c|c|c|c|c|}
\hline Domínio & IMC & $\mathrm{n}$ & Média \pm DP & $\mathbf{p}^{*}$ \\
\hline \multirow[t]{4}{*}{ Físico } & Magreza & 3 & $66,67 \pm 12,54$ & 0,015 \\
\hline & Eutrofia & 21 & $64,97 \pm 14,09$ & \\
\hline & Sobrepeso & 23 & $51,86 \pm 20,54$ & \\
\hline & Obesidade & 32 & $49,00 \pm 20,06$ & \\
\hline \multirow[t]{4}{*}{ Psíquico } & Magreza & 3 & $66,67 \pm 8,33$ & 0,148 \\
\hline & Eutrofia & 21 & $61,90 \pm 13,84$ & \\
\hline & Sobrepeso & 23 & $56,70 \pm 16,08$ & \\
\hline & Obesidade & 32 & $52,21 \pm 20,30$ & \\
\hline \multirow[t]{4}{*}{ Social } & Magreza & 3 & $63,89 \pm 4,81$ & 0,372 \\
\hline & Eutrofia & 21 & $70,24 \pm 12,79$ & \\
\hline & Sobrepeso & 23 & $70,29 \pm 17,19$ & \\
\hline & Obesidade & 32 & $64,58 \pm 18,33$ & \\
\hline \multirow[t]{4}{*}{ Ambiental } & Magreza & 3 & $68,75 \pm 3,13$ & 0,178 \\
\hline & Eutrofia & 21 & $65,77 \pm 11,07$ & \\
\hline & Sobrepeso & 23 & $62,23 \pm 11,07$ & \\
\hline & Obesidade & 32 & $58,11 \pm 13,93$ & \\
\hline Domínio & Classificação do risco cardiovascular classificado pela CC & n & Média \pm DP & $\mathbf{p}^{*}$ \\
\hline \multirow[t]{2}{*}{ Físico } & Sem risco cardiovascular & 26 & $59,48 \pm 20,80$ & 0,078 \\
\hline & Com risco cardiovascular & 53 & $52,43 \pm 18,73$ & \\
\hline \multirow[t]{2}{*}{ Psíquico } & Sem risco cardiovascular & 26 & $58,33 \pm 15,41$ & 0,637 \\
\hline & Com risco cardiovascular & 53 & $55,82 \pm 18,55$ & \\
\hline \multirow[t]{2}{*}{ Social } & Sem risco cardiovascular & 26 & $68,59 \pm 15,15$ & 0,811 \\
\hline & Com risco cardiovascular & 53 & $67,30 \pm 17,05$ & \\
\hline \multirow{2}{*}{ Ambiental } & Sem risco cardiovascular & 26 & $62,74 \pm 10,11$ & 0,810 \\
\hline & Com risco cardiovascular & 53 & $61,26 \pm 13,52$ & \\
\hline Domínio & Classificação do risco de excesso de peso pela CP & $\mathbf{n}$ & Média \pm DP & $\mathbf{p}^{*}$ \\
\hline \multirow[t]{2}{*}{ Físico } & Sem risco excesso de peso & 55 & $57,08 \pm 19,48$ & 0,060 \\
\hline & Com risco excesso de peso & 24 & $49,40 \pm 19,15$ & \\
\hline \multirow[t]{2}{*}{ Psíquico } & Sem risco excesso de peso & 55 & $58,11 \pm 16,50$ & 0,256 \\
\hline & Com risco excesso de peso & 24 & $53,30 \pm 19,62$ & \\
\hline \multirow[t]{2}{*}{ Social } & Sem risco excesso de peso & 55 & $68,94 \pm 15,99$ & 0,293 \\
\hline & Com risco excesso de peso & 24 & $64,93 \pm 17,20$ & \\
\hline \multirow{2}{*}{ Ambiental } & Sem risco excesso de peso & 55 & $63,35 \pm 11,23$ & 0,102 \\
\hline & Com risco excesso de peso & 24 & $58,07 \pm 14,48$ & \\
\hline
\end{tabular}

*: Teste Mann-Whitney; CC: Circunferência da cintura; CP: Circunferência do pescoço; IMC: Índice de Massa Corporal 
Tabela IV - Correlação dos domínios de qualidade de vida com as variáveis de idade, Índice de Massa Corporal, Circunferência da Cintura e Circunferência do Pescoço de adultos e idosos das Unidades Básicas de Saúde dos municípios do Vale do Taquari. Rio Grande do Sul, Brasil, 2017.

\begin{tabular}{lcccccccc}
\hline \multirow{2}{*}{ Variável } & \multicolumn{2}{c}{ Domínio Físico } & \multicolumn{2}{c}{ Domínio Psíquico } & \multicolumn{2}{c}{ Domínio Social } & \multicolumn{2}{c}{ Domínio Ambiental } \\
& $\mathbf{r}$ & $\mathbf{p}$ & $\mathbf{r}$ & $\mathbf{p}$ & $\mathbf{r}$ & $\mathbf{p}$ & $\mathbf{r}$ & $\mathbf{p}$ \\
\hline Idade & 0,268 & 0,017 & 0,409 & $\mathbf{p}<0,01$ & 0,359 & 0,001 & 0,328 & 0,003 \\
IMC $\mathbf{~ g g / m} \mathbf{m}^{\mathbf{2}}$ & $-0,292$ & 0,009 & $-0,257$ & 0,022 & $-0,215$ & 0,058 & $-0,267$ & 0,018 \\
$\mathbf{C C} \mathbf{( c m )}$ & $-0,139$ & 0,222 & $-0,105$ & 0,356 & $-0,002$ & 0,989 & $-0,175$ & 0,122 \\
$\mathbf{C P} \mathbf{( c m )}$ & $-0,115$ & 0,312 & $-0,028$ & 0,809 & 0,016 & 0,891 & $-0,069$ & 0,545 \\
\hline
\end{tabular}

r: Teste de correlação de Pearson; IMC: Índice de Massa Corporal; $\mathrm{Kg} / \mathrm{m}^{2}$ : quilogramas por metro quadrado; CC: Circunferência da cintura; CP: Circunferência do pescoço; $\mathrm{cm}$ : centímetros

Tabela V - Comparação entre pacientes deprimidos e não deprimidos considerando seu estado nutricional a partir de dados de Índice de Massa Corporal, Circunferência da Cintura e Circunferência do Pescoço de adultos e idosos de Unidades Básicas de Saúde dos municípios do Vale do Taquari. Rio Grande do Sul, Brasil, 2017.

\begin{tabular}{llccccc}
\hline \multirow{2}{*}{ Variável } & Resposta & \multicolumn{3}{c}{ Tipo de paciente } & \\
& & Deprimido & Não deprimido & $\mathbf{p}^{*}$ \\
\hline Classificação IMC & Magreza & $\mathbf{n}$ & $\mathbf{\%}$ & $\mathbf{n}$ & $\mathbf{\%}$ & \\
& Eutrofia & - & - & 3 & $7,3 \%$ & 0,094 \\
& Sobrepeso & 7 & $18,4 \%$ & 14 & $34,1 \%$ & \\
& Obesidade & 12 & $31,6 \%$ & 11 & $26,8 \%$ & \\
Classificação CC & Sem risco cardiovascular & 19 & $50,0 \%$ & 13 & $31,7 \%$ & \\
& Com risco cardiovascular & 29 & $23,7 \%$ & 17 & $41,5 \%$ & 0,093 \\
Classificação & Sem risco excesso de peso & 25 & $65,8 \%$ & 30 & $73,2 \%$ & 0,476 \\
CP & Com risco excesso de peso & 13 & $34,2 \%$ & 11 & $26,8 \%$ & \\
\hline
\end{tabular}

*: Teste Qui-quadrado; CC: Circunferência da cintura; CP: Circunferência do pescoço

\section{DISCUSSÃO}

A partir dos dados coletados no presente estudo, percebeu-se que a maior parte dos participantes se apresentou com sobrepeso ou obesidade. Entretanto, a melhor média para QV foi verificada entre os participantes classificados com magreza e eutrofia. O IMC demonstrou correlação inversa em relação aos domínios de QV, os homens, idosos e os pacientes não deprimidos obtiveram médias superiores em todos os domínios de QV. A QV é relacionada às mudanças de costumes, intervenção, política e atitudes que contribuam para melhorias na saúde da população. Portanto, cabe salientar que a promoção da saúde se relaciona a esses conceitos, pois o ambiente influencia nos hábitos de uma comunidade ${ }^{(18)}$.

Referente ao estado nutricional, observou-se que a maior parte dos participantes da atual pesquisa recebeu a classificação de obesidade, seguido pelo sobrepeso. Esses achados corroboram um estudo realizado com 11 idosas, em uma instituição de ensino superior privada da cidade de Itabuna, na Bahia, no qual $42,32 \%$ das participantes apresentaram peso adequado e $46,15 \%$ sobrepeso $^{(19)}$. Em um estudo com 155 adultos, realizado em uma UBS, verificou-se que o excesso de peso estava presente em $36,1 \%$ dos participantes e a obesidade em $29,0 \%{ }^{(20)}$. Em outro estudo com 156 idosos participantes de um grupo de terceira idade, foi observado que o estado nutricional de $43,6 \%$ dos idosos estava com sobrepeso ou obesidade ${ }^{(21)}$.

Esses resultados reiteram a quarta colocação do Brasil no mundo em prevalência de obesidade. A situação em questão está associada a diversas enfermidades cardiovasculares, por isso é importante destacar que essa condição pode ser evitada por meio de ações de prevenção de fatores de risco comportamentais, como dietas inadequadas, sedentarismo e uso exagerado de álcool e/ou tabagismo ${ }^{(22)}$. Nesse sentido, a PNaPS (Política Nacional de Promoção da Saúde) destaca a importância da promoção da saúde, da segurança alimentar e nutricional, da redução da pobreza, da inclusão social e da segurança dos direitos humanos à alimentação adequada e saudável como parte das possíveis estratégias ${ }^{(23)}$. 
No atual estudo, $67,1 \%$ dos participantes apresentaram risco cardiovascular pela classificação da CC, resultado inferior ao observado em um estudo realizado em Itabuna, na Bahia, com participantes do sexo feminino. Nesse estudo, $80,76 \%$ dos participantes apresentaram risco de desenvolvimento de doenças cardiovasculares ${ }^{(19)}$, resultados estes superiores ao estudo que encontrou $54,8 \%$ dos participantes com CC aumentada ${ }^{(20)}$. Conforme evidenciado em um estudo com 120 mulheres de 20 a 49 anos, residentes no município de São José de Ribamar, Maranhão, verificou que $55 \%$ das participantes apresentaram risco aumentado para desenvolvimento de doenças cardiovasculares, mediante resultados dos dados da $\mathrm{CC}^{(24)}$. Sabendo que a doença cardiovascular é considerada uma das principais causa de óbitos no Brasil, correspondendo a $30 \%$ das mortes, faz-se necessária a implementação de estratégias de saúde desenvolvidas na atenção primária e secundária, com foco na prevenção dos fatores de risco(25).

Em relação aos domínios de qualidade de vida dos adultos e idosos, verificou-se que as médias foram: social 67,72 , ambiental 61,75 , psíquico 56,65 e físico 54,75 . Desse modo, elas foram inferiores às encontradas em um estudo com 930 adultos e idosos atendidos na UBS de Belo Horizonte, onde se observaram médias dos domínios social de 68,2 , ambiental de 52,4, psíquico de 66,5 e físico de 63,0 ${ }^{(26)}$. Por fim, em um estudo realizado com adultos e idosos beneficiários de programas de transferência de renda, moradores do interior de São Paulo, as médias encontradas, quando comparadas ao atual estudo, são inferiores em todos os domínios: social $(53,4)$, ambiental $(43,0)$, psíquico $(54,9)$ e físico $(54,1)^{(8)}$.

No presente estudo, observou-se que o sexo masculino obteve média significativamente superior no domínio ambiental em relação às mulheres, demonstrando que a satisfação dos homens em relação à segurança, recursos financeiros, transporte e aquisição de bens é superior ${ }^{(27)}$, podendo estar relacionado com a maior valorização do homem o que diz respeito a estas questões. Já em outro estudo, constatou-se diferença estatisticamente significativa apenas para o domínio psicológico $(p \leq 0,002)^{(26)}$, com os homens apresentando maior escore em relação a esse domínio. Isso pode ser justificado pelo fato de as mulheres terem uma percepção da depressão duas vezes maior que os homens, o que pode ser influenciado por diferenças fisiológicas e hormonais, baixa escolaridade e renda, cultura, além de diferentes maneiras de lidar com momentos de estresse ${ }^{(28)}$.

Em relação às médias dos domínios físico e psíquico, verificou-se média significativamente superior entre os pacientes não deprimidos em relação aos deprimidos na presente pesquisa. Um estudo desenvolvido com 1.560 adultos, na cidade de Pelotas, Rio Grande do Sul, utilizando o método de avaliação do Mini-International Neuropsychiatric Interview (MINI) para diagnóstico de depressão e Medical Outcomes Study Short-form General Health Survery (SF-36) para avaliação da qualidade de vida, encontrou resultados semelhantes aos aqui expostos, com médias superiores e significativas entre as pessoas sem o diagnóstico de depressão, no domínio físico e no social ${ }^{(29)}$. A depressão é vista como uma das principais causas de incapacitação no mundo, diminuindo o funcionamento físico, pessoal e social, além de prejudicar os quadros clínicos, envolvendo as cardiopatias, diabetes, obesidade e problemas oncológicos ${ }^{(30)}$. Assim, é de extrema importância o incentivo à promoção da saúde, com o intuito de melhorar a qualidade de vida da população, privilegiando a criação de estratégias sociais e de ações intersetoriais, com equipes multidisciplinares ${ }^{(7)}$.

Quanto à comparação entre os domínios de qualidade de vida e de faixa etária, os idosos apresentam média significativamente superior nos domínios ambiental, psíquico e físico quando contrapostos aos adultos. Em outro estudo, averiguou-se que, comparados a adultos jovens com idades entre 18 e 39 anos, a população idosa apresentou média superior apenas no domínio ambiental; quando comparados os resultados de adultos com idade entre 40 e 59 anos, os idosos apresentaram média superior em todos os domínios ${ }^{(26)}$. Em um estudo com 77 idosos institucionalizados, foi evidenciado que os idosos mais jovens, que participam de atividades diárias, apresentaram, em geral, melhor percepção na QV, o que pode estar relacionado aos cuidados e à atenção que eles recebem ${ }^{(31)}$.

Para garantir o bem-estar da população idosa, o SUS organiza estratégias de atenção à saúde da melhor idade a partir do modelo de atenção integral, que destaca a importância e o potencial do trabalho em rede, com vistas ao cuidado à pessoa idosa e à adequação do atendimento às suas necessidades. Estratégias como essas são de extrema valia, pois se baseiam no aumento da expectativa de vida. O envelhecimento da população brasileira trouxe demandas que envolvem respostas das políticas sociais, implicando planejamento de novas formas de cuidado, especialmente nos casos prolongados e na atenção domiciliar. A combinação de subsídios econômicos, alimentação adequada e intervenções de saúde propiciam bons resultados para a adesão dos idosos aos programas de saúde ${ }^{(32)}$. O processo de envelhecimento saudável melhora com uma boa QV, proporcionando diminuição ao risco de doenças, bom desempenho físico, estado ativo para as atividades diárias e bom funcionamento mental ${ }^{(33)}$. Em contrapartida, a população de adultos valoriza os fatores relacionados à atividade laboral e a família, mostrando preocupação com as questões financeiras, formação familiar e a busca de conquista de bens materiais, focando na estabilidade 
financeira, situações diárias relacionadas com a falta de tempo para realizar atividades de lazer e insatisfação financeira, diferente dos idosos. Essas questões que podem influenciar na percepção da QV da população adulta(34).

O surgimento da depressão está diretamente relacionado à rotina diária, aos fatores psicossociais, emotivos e econômicos. A participação dos idosos em atividades comunitárias é de extrema relevância, pois o não engajamento em uma comunidade é uma das possíveis causas dos casos diagnosticados de depressão ${ }^{(35)}$.

A magreza e a eutrofia apresentaram média significativamente superior no domínio físico, resultado semelhante a um estudo realizado com 370 idosos no município do Sudoeste do Brasil, onde se verificou que os idosos com excesso de peso apresentaram escores significativamente inferiores no domínio físico, quando comparado aos que não apresentam excesso de peso( ${ }^{(36)}$. Assim como em outro estudo, com 156 idosos, no qual se observou que a média do domínio físico foi significativamente menor entre os indivíduos com IMC elevado ${ }^{(21)}$. O domínio físico inclui questões referentes à dor e desconforto, energia e fadiga, sono e descanso, mobilidade, atividades diárias, uso de medicação/tratamento e aptidão para o trabalho ${ }^{(37)}$, sabendo disso, pressupõe-se que o excesso de peso tenha uma considerável interferência nestas questões, influenciando negativamente na capacidade funcional destes indivíduos ${ }^{(25)}$.

Em relação ao diagnóstico de depressão e o estado nutricional, observou-se que $50 \%$ dos participantes deprimidos foram classificados com obesidade e 31,6\% com sobrepeso. Em um estudo que analisou 49.025 indivíduos adultos com e sem depressão, evidenciou-se que os depressivos possuíam maior prevalência em todos os comportamentos considerados prejudiciais à saúde. Analisando a associação entre depressão e alimentação, verificou-se que todos os indicadores ligados à alimentação não saudável foram mais predominantes nos indivíduos depressivos ${ }^{(38)}$. Esse resultado corrobora, portanto, o presente estudo, pois os participantes com depressão obtiveram menor QV.

A depressão pode facilitar o desenvolvimento da obesidade devido às alterações nos hábitos alimentares e à diminuição da atividade física. Da mesma forma, a obesidade pode influenciar no surgimento da depressão, devido à assimilação negativa da imagem corporal(39). Sendo assim, as intervenções em saúde possuem como objetivo os problemas e as necessidades da população, portanto focam no desenvolvimento de ações e de serviços que envolvam a prevenção do adoecimento, abrangendo as condições de vida e estimulando as escolhas saudáveis ${ }^{(5)}$.

Como limitação deste estudo, é necessário destacar que os resultados obtidos podem não ser representativos para toda a população atendida nas Unidades Básicas de Saúde, uma vez que a amostra estudada foi de pacientes com atendimento psicológico. Portanto, percebe-se a necessidade de mais estudos com a população atendida pelo serviço de psicologia da rede pública, com o intuito de promover saúde, qualidade de vida e bem-estar, com atendimento integral respeitando as especificidades de cada indivíduo na construção de projetos terapêuticos ${ }^{(23)}$.

\section{CONCLUSÃO}

As melhores percepções sobre os domínios de qualidade de vida foram observadas entre homens, idosos e sem diagnóstico de depressão. Verificou-se correlação direta entre a idade e todos os domínios e que os participantes depressivos eram, em sua maioria, obesos, com risco cardiovascular e sem risco de excesso de peso.

\section{CONFLITOS DE INTERESSE}

As autoras afirmam que não houve conflitos de interesse na execução desta pesquisa.

\section{CONTRIBUIÇÕES}

Aline Rodrigues Godoy e Fernanda Scherer Adami: Elaboração e delineamento do estudo; aquisição, análise e interpretação dos dados; redação do manuscrito.

\section{REFERÊNCIAS}

1. Sezini AM, Gil CSGC. Nutrientes e depressão. Vita et Sanitas [Internet]. 2014;8(1):39-57. [acesso em 2017 Out 31]. Disponível em: http://www.fug.edu.br/revista/index.php/VitaetSanitas/article/view/29/21

2. Rodrigues LR, Tavares DMS, Silveira FCO, Dias FA, Martins NPF. Qualidade de vida, indicativo de depressão e número de morbidades de idosos da zona rural. Rev Enferm Atenção Saúde [Internet]. 2015;4(2):33-44. [acesso em 2017 Nov 14]. Disponível em: http://seer.uftm.edu.br/revistaeletronica/index.php/enfer/article/ view/1122/pdf 
3. Organização Pan-Americana da Saúde. Folha informativa - Depressão [Internet]. 2018; [acesso em 2018 Set 07]. Disponível em: https://www.paho.org/bra/index.phpoption=com_content\&view=article\&id=5635:folhainformativa-depressao\&ltemid $=822$

4. Organização Pan-Americana da Saúde. Com depressão no topo da lista de causas de problemas de saúde, OMS lança a campanha "Vamos conversar" [Internet]. 2017; [acesso em 2018 Set 07]. Disponível em: https:// www.paho.org/bra/index.php?option=com_content\&view=article\&id=5385:com-depressao-no-topo-da-lista-decausas-de-problemas-de-saude-oms-lanca-a-campanha-vamos-conversar\&ltemid=839

5. Ministério da Saúde (BR). Secretaria de Vigilância em Saúde. Política Nacional de Promoção da Saúde. 3a ed. Brasília: Ministério da Saúde; 2010.

6. Máximo GC. Aspectos sociodemográficos da depressão e utilização de serviços de saúde no Brasil [tese]. Belo Horizonte: Universidade Federal de Minas Gerais; 2010. [acesso em 2018 Set 07]. Disponível em: http:// www.bibliotecadigital.ufmg.br/dspace/bitstream/handle/1843/AMSA-8BCKKK/geovane_maximo_versao_ out_2010.pdf?sequence $=1$

7. Garcia PT, Reis RS, organizadores. Redes de atenção à saúde: Rede de Atenção Psicossocial - RAPS [Internet]. São Luís: EDUFMA; 2018. [acesso em 2017 Nov 05]. Disponível em: http://www.unasus.ufma.br/ site/files/livros_isbn/isbn_redes06.pdf

8. Ribas-Prado MC, Calais SL, Cardoso HF. Stress, Depressão e qualidade de vida em beneficiários de programas de transferência de renda. Interação Psicol [Internet]. 2016;20(3):330-40. [acesso em 2017 Nov 05]. Disponível em: http://revistas.ufpr.br/psicologia/article/view/35133/31288

9. Lourenço LMP. A Influência da pobreza no estado de saúde da população idosa de Paredes de Coura [dissertação]. Portugal: Instituto Politécnico de Viana do Castelo; 2013. [acesso em 2018 Set 07]. Disponível em: http://repositorio.ipvc.pt/bitstream/20.500.11960/1204/3/Liliana_Lourenco.pdf

10. Guerra A, Silva A, Sousa C, Ferreira L, Carvalho H, Silva S. A Componente mental: um aspeto positivo da qualidade de vida de uma população. Rev Port Enferm Saúde Mental [Internet]. 2017;5:75-80. [acesso em 2018 Set 07]. Disponível em: http://dx.doi.org/10.19131/rpesm.0171

11. Politi R. Desigualdade na utilização de serviços de saúde entre adultos: uma análise dos fatores de concentração da demanda. Econ Aplic [Internet]. 2014;18(1):117-37. [acesso em 2018 Set 07]. Disponível em: http://www.scielo.br/scielo.php?script=sci_arttext\&pid=S1413-80502014000100006

12. Sousa AAD, Martins AMEBL, Silveira MF, Coutinho WLM, Freitas DA, Vasconcelos EL et al. Qualidade de vida e incapacidade funcional entre idosos cadastrados na estratégia de saúde da família. $A B C S$ Health Sci [Internet]. 2018;43(1):14-24. [acesso em 2018 Set 07]. Disponível em: http://docs.bvsalud.org/ biblioref/2018/06/883996/abcs-health-pdf.pdf

13. Pereira RJ, Cotta RMM, Franceschini SCC, Ribeiro RCL, Sampaio RF, Priore SE et al. Contribuição dos domínios físico, social, psicológico e ambiental para a qualidade de vida global de idosos. Rev Psiquiatr Rio Gd Sul [Internet]. 2006;28(1):27-38. [acesso em 2018 Set 07]. Disponível em: http://www.scielo.br/pdf/rprs/ v28n1/v28n1a05.pdf

14. Pereira KCR, Alvarez AM, Traebert JL. Contribuição das condições sóciodemográficas para a percepção da qualidade de vida em idosos. Rev Bras Geriatr Gerontol [Internet]. 2011;14(1):85-95. [acesso em 2018 Set 07]. Disponível em: http://www.scielo.br/pdf/rbgg/v14n1/a10v14n1.pdf

15. Interdonato GC, Greguol M. Qualidade de vida percebida por indivíduos fisicamente ativos e sedentários. Rev Bras Ciênc Mov [Internet]. 2010;18(1):61-7. [acesso em 2018 Set 07]. Disponível em: https://portalrevistas. ucb.br/index.php/RBCM/\%20article/viewFile/1379/1727

16. World Health Organization. Physical status the use and interpretation of anthropometrics: report of a World Health Organization Expert Committee. Genebra: WHO; 1995.

17. Mussoi TD. Avaliação nutricional na prática clínica: da gestação ao envelhecimento. Rio de Janeiro: Guanabara Koogan; 2014.

18. Oliveira ERA, Garcia AL, Gomes MJ, Bittar TO, Pereira AC. Gênero e qualidade de vida percebida - estudo com professores da área de saúde. Ciênc Saúde Colet [Internet]. 2012;17(3):741-47. [acesso em 2018 Set 08]. Disponível em: http://www.scielo.br/scielo.php?script=sci_arttext\&pid=S1413-81232012000300021 
19. Santos RKF, Vital AVD. Perfil nutricional de idosos - relação obesidade e circunferência da cintura após sessenta anos. Revista Saúde.com [Internet]. 2014;10(3):254-62. [acesso em 2018 Set 08]. Disponível em: http://periodicos2.uesb.br/index.php/rsc/article/view/310/249

20. Frizon V, Boscaini C. Circunferência do pescoço, fatores de risco para doenças cardiovasculares e consumo alimentar. Rev Bras Cardiol [Internet]. 2013;26(6):426-34. [acesso em 2018 Set 08]. Disponível em: file://C:/ Users/730546923/Downloads/v26n6a03.pdf

21. Adami FS, Feil CC, Dal Bosco SM. Estado nutricional relacionado à qualidade de vida em idosos. Int $\mathrm{J}$ Aging Hum Dev. 2015;12(1):28-40.

22. Rocha RM, Martins WA, editores. Manual de prevenção cardiovascular. Rio de Janeiro: Sociedade de Cardiologia do Estado do Rio de Janeiro; 2017.

23. Ministério da Saúde (BR). Secretaria de Vigilância em Saúde. Secretaria de Atenção à Saúde. Política Nacional de Promoção da Saúde PNPS: revisão da Portaria MS/GM nº 687, de 30 de março de 2006. Brasília: Ministério da Saúde; 2015.

24. Moraes KD, Araújo AP, Santos AF, Barbosa JMA, Martins MLB. Correlação entre o índice de massa corporal e indicadores antropométricos de risco cardiovascularizar em mulheres. Rev Pesq Saúde [Internet]. 2015;16(3):175-81. [acesso em 2018 Set 08]. Disponível em: http://www.periodicoseletronicos.ufma.br/index. php/revistahuufma/article/view/4517

25. Hirata RP, Cerra JC, Macedo CR, Favareto J, Leitão FSS Filho, Oliveira LVF. Prevalência de obesidade e hipertensão arterial em uma população de motoristas profissionais rodoviários interestaduais de ônibus. Conscientiae Saúde [Internet]. 2011;10(3):494-99. [acesso em 2018 Set 08]. Disponível em: http://www. redalyc.org/pdf/929/92920013012.pdf

26. Almeida-Brasil CC, Silveira MR, Silva KR, Lima MG, Faria CDCM, Cardoso CL et al. Qualidade de vida e características associadas: aplicação do WHOQOL-BREF no contexto da Atenção Primária à Saúde. Ciênc Saúde Coletiva [Internet]. 2017;22(5):1705-16. [acesso 2018 Set 08]. Disponível em: http://www.scielo.br/ scielo.php?pid=S1413-81232017002501705\&script=sci_abstract

27. Souza DP, Melo TS, Reis LA, Lima PV. Qualidade de vida em idosos portadores de hipertensão arterial e diabetes mellitus. Rev Psicol [Internet]. 2016;10(31):56-68. [acesso em 2018 Out 15]. Disponível em: https:// idonline.emnuvens.com.br/id/article/view/547/733

28. Gonçalves AMC, Teixeira MTB, Gama JRA, Lopes CS, Silva GA, Gamarra CJ et al. Prevalência de depressão e fatores associados em mulheres atendidas pela Estratégia de Saúde da Família. J Bras Psiquiatr [Internet]. 2018;67(2):101-9. [acesso 2018 Out 15]. Disponível em: https://repositorio.ufjf.br/jspui/bitstream/ufff/7531/1/ Preval\%C3\%Aancia\%20de\%20depress\%C3\%A30\%20e\%20fatores\%20associados\%20em\%20mulheres.pdf

29. Lopez MRA, Ribeiro JP, Ores LC, Jansen K, Souza LDM, Pinheiro RT et al. Depressão e qualidade de vida em jovens de 18 a 24 anos no sul do Brasil. Rev Psiquiatr Rio Gd Sul [Internet]. 2011;33(2):103-8. [acesso 2018 Set 08]. Disponível em: http://www.scielo.br/pdf/rprs/v33n2/1338.pdf

30. Loosli L, Pizeta FA, Loureiro SR. Escolares que convivem com a depressão materna recorrente: diferenças entre os sexos. Psicol Teor Pesqui [Internet]. 2016;32(3):1-10. [acesso 2018 Out 15]. Disponível em: http:// www.scielo.br/pdf/ptp/v32n3/1806-3446-ptp-32-03-e32322.pdf

31. Vitorino LM, Vianna LAC, Paskulin LMG. Qualidade de vida de idosos em instituição de longa permanência. Rev Latinoam Enferm [Internet]. 2012;20(6). [acesso 2018 Set 08]. Disponível em: http://www.scielo.br/pdf/ rlae/v20n6/pt_22.pdf

32. Ministério da Saúde (BR). Secretaria de Atenção à Saúde. Diretrizes para o cuidado das pessoas idosas no SUS: proposta de modelo de atenção integral. Brasília: Ministério da Saúde; 2014.

33. Almeida LFF, Gomes IS, Salgado SML, Freitas EL, Almeida RWS, Oliveira WC et al. Promoção da saúde, qualidade de vida e envelhecimento - a experiência do projeto "Em Comum-Idade: uma proposta de ações integradas para a promoção da saúde de idosos das comunidades de Viçosa-MG". Rev Elo - Diálogo Extensão [Internet]. 2014;3(2):71-80. [acesso em 2018 Set 08]. Disponível em: https://periodicos.ufv.br/ojs/ elo/article/view/1027/575 
34. Sonati JG, Vilarta R, Maciel ES, Modeneze DM, Vilela GB Jr., Lazari VO et al. Análise comparativa da qualidade de vida de adultos e idosos envolvidos com a prática regular de atividade física. Rev Bras Geriatr Gerontol [Internet]. 2014;17(4):731-39. [acesso em 2018 Out 16]. Disponível em: http://www.scielo.br/pdf/ rbgg/v17n4/1809-9823-rbgg-17-04-00731.pdf

35. Magalhães JM, Carvalho AMB, Carvalho SM, Alencar DC, Moreira WC, Parente ACM. Depressão em idosos na estratégia saúde da família: uma contribuição para a atenção primária. REME Rev Min Enferm [Internet]. 2016;20(e947):1-6. [acesso em 2018 Set 08] Disponível em: http://www.reme.org.br/artigo/detalhes/1080

36. Tavares DMS, Bolina AF, Dias FA, Ferreira PCS, Santos NMF. Excesso de peso em idosos rurais: associação com as condições de saúde e qualidade de vida. Ciênc Saúde Colet [Internet]. 2018;23(3):913-22. [acesso em 2018 Out 16]. Disponível em: https://www.scielosp.org/article/ssm/content/raw/?resource_ssm_path=/ media/assets/csc/v23n3/1413-8123-csc-23-03-0913.pdf.

37. Braccialli LMP, Bagagi PS, Sankako AN, Araújo RCT. Qualidade de vida de cuidadores de pessoas com necessidades especiais. Rev Bras Educ Espec [Internet]. 2012;18(1):113-26. [acesso em 2018 Out 16]. Disponível em: http://www.scielo.br/scielo.php?script=sci_arttext\&pid=\$1413-65382012000100008.

38. Borros MBA, Lima MG, Azevedo RCS, Medina LBP, Lopes CS, Menezes PR et al. Depressão e comportamentos de saúde em adultos brasileiros - PNS 2013. Rev Saúde Publica [Internet]. 2017;51(1):1s-10s. [acesso em 2018 Set 08]. Disponível em: http://www.redalyc.org/articulo. oa?id=67251395009

39. Maria CC, Yaegashi SFR. Os traços de personalidade associados ao desenvolvimento da obesidade. Rev Bras Obesid Nutri Emagrec [Internet]. 2016;10(56):74-92. [acesso em 2018 Set 08]; Disponível em: http:// www.rbone.com.br/index.php/rbone/article/view/411/380

\author{
Endereço do primeiro autor: \\ Aline Rodrigues Godoy \\ Rua Daniel Ahne, 99 \\ Bairro: Centro \\ CEP: 95948-000 - Travesseiro - RS - Brasil \\ E-mail: alyrgodoy@yahoo.com.br \\ Endereço para correspondência: \\ Fernanda Scherer Adami \\ Universidade do Vale do Taquari - UNIVATES \\ Av. Avelino Talini, 171 \\ Bairro: Universitário \\ CEP: 95914-014 - Lajeado - RS - Brasil \\ E-mail: fernandascherer@univates.br
}

Como citar: Godoy AR, Adami FS. Estado nutricional e qualidade de vida em adultos com depressão. Rev Bras Promoç Saúde. 2019;32:7354. 